\title{
Stability of Graphs
}

\author{
Bünyamin Demir, Ali Deniz and Şahin Koçak \\ Anadolu University \\ Department of Mathematics \\ Yunusemre Kampusu, 26470, Eskiehir, Turkey \\ bdemir@anadolu.edu.tr, adeniz@anadolu.edu.tr, skocak@anadolu.edu.tr \\ Submitted: Sep 1, 2008; Accepted: Feb 11, 2009; Published: Feb 20, 2009 \\ Mathematics Subject Classification: 05C10, 51F99
}

\begin{abstract}
Positively weighted graphs have a natural intrinsic metric. We consider finite, positively weighted graphs with a positive lower bound for their minimal weights and show that any two such graphs, which are close enough with respect to the Gromov-Hausdorff metric, are equivalent as graphs.
\end{abstract}

\section{Introduction}

We consider finite, connected graphs with positive weights assigned to edges. We allow loops and multiple edges, but we exclude vertices of degree 2. (The meaning of this restriction will be clear in the sequel.) We will call such graphs admissible weighted graphs. One can define a natural metric on an admissible weighted graph [4] and then the Gromov-Hausdorff distance between such graphs makes sense. We will prove the following theorem, which could be interpreted as a stability property for graphs:

Theorem 1. Let $G$ be an admissible weighted graph with minimal weight $\geq r>0$. If $H$ is any other admissible weighted graph with minimal weight $\geq r$, which is close enough (in the Gromov-Hausdorff metric) to $G$, then $H$ is equivalent to $G$ as a graph. (i.e. there are bijections between vertices and between edges respecting the incidence relations.)

\section{Metric Geometry for Graphs}

For the general theory of length spaces, intrinsic metrics and Gromov-Hausdorff metric we refer to [4], and we only briefly define the relevant notions so far as we need them for graphs.

If $(X, d)$ is a complete metric space, then the metric is said to be strictly intrinsic iff for any two points $x, y \in X$ there exists a midpoint $z \in X$ (i.e. a point $z$ with 
$\left.d(x, z)=d(z, y)=\frac{1}{2} d(x, y)\right)$. Equivalently, for any two points $x, y \in X$ and $\varepsilon>0$ there must exist a finite sequence of points $x=x_{0}, x_{1}, x_{2}, \ldots, x_{k}=y$ such that consecutive points are $\varepsilon$-close (i.e. $d\left(x_{i}, x_{i+1}\right) \leq \varepsilon$ for $\left.i=0,1, \ldots, k-1\right)$ and $\sum_{i=0}^{k-1} d\left(x_{i}, x_{i+1}\right)=d(x, y)$.

Now let us given an admissible weighted graph $G=(V, E, I, \omega)$ where $V$ is the set of vertices, $E$ is the set of edges, $I: \partial E \rightarrow V$ is the identification map ( $\partial E$ is the set of end-points of the edges) and $\omega: E \rightarrow \mathbb{R}^{+}$a positive-valued weight map. We can consider $G$ as a compact, connected topological space by making the appropriate identifications on the disjoint union of the edges.

On any edge $e \in E$ of a graph $G=(V, E, I, \omega)$, the weight $\omega(e)$ defines a natural metric, making the (unglued) edge isometric with the interval $[0, \omega(e)] \subset \mathbb{R}$ and we denote this metric by $d_{e}$. Now, given any pair of points $x, y \in G$, consider the sequences $x_{0}=$ $x, x_{1}, x_{2}, \ldots, x_{n}=y$ such that the consecutive points $x_{i}, x_{i+1}$ lie on the same edge, say $e_{i}$, and define

$$
d(x, y)=\inf \left\{\sum_{i=0}^{n-1} d_{e_{i}}\left(x_{i}, x_{i+1}\right)\right\} .
$$

(For simplicity we abuse notation by denoting identified points with the same symbol. Loops do not do any harm as the infimum is taken, but for definiteness one could also take as $d_{e_{i}}\left(x_{i}, x_{i+1}\right)$ the "length" of the shorter subsegment if $x_{i}, x_{i+1}$ lie on a loop and one of them is the vertex.) It can be shown that $d$ is a complete metric (inducing the right topology on $G$ ) which is strictly intrinsic.

Given two compact subspaces $X, Y$ of a metric space $Z$, the Hausdorff distance $d_{H}(X, Y)$ is the infimum of $\varepsilon$, for which $X$ lies in the $\varepsilon$-neighborhood of $Y$ and $Y$ lies in the $\varepsilon$-neighborhood of $X$. If $X$ and $Y$ are any compact metric spaces, we can consider possible isometric copies $X^{\prime}, Y^{\prime}$ lying in a metric space $Z^{\prime}$ and compute $d_{H}\left(X^{\prime}, Y^{\prime}\right)$ inside $Z^{\prime}$. The Gromov-Hausdorff distance $d_{G H}(X, Y)$ is the infimum of all such $d_{H}\left(X^{\prime}, Y^{\prime}\right)$. The Gromov-Hausdorff distance defines a metric on the space of isometry classes of compact metric spaces.

A useful notion is that of an $\varepsilon$-isometry between metric spaces. For metric spaces $\left(X, d_{X}\right),\left(Y, d_{Y}\right)$ and $\varepsilon>0$, a function $f: X \rightarrow Y$ is called an $\varepsilon$-isometry if distortion $(f) \leq$ $\varepsilon$ and $f(X)$ is an $\varepsilon$-net in $Y$, whereby

$$
\operatorname{distortion}(f)=\sup _{x_{1}, x_{2} \in X}\left|d_{Y}\left(f\left(x_{1}\right), f\left(x_{2}\right)\right)-d_{X}\left(x_{1}, x_{2}\right)\right| .
$$

( $\varepsilon$-net condition means that, for any $y \in Y$ there exists $x \in X$ with $d_{Y}(f(x), y) \leq \varepsilon$.) The following proposition relates Gromov-Hausdorff distance to $\varepsilon$-isometry [4].

Proposition 1. Let $X$ and $Y$ be two metric spaces and $\varepsilon>0$. Then,

1. If $d_{G H}(X, Y)<\varepsilon$, then there exists a $2 \varepsilon$-isometry from $X$ to $Y$.

2. If there exists an $\varepsilon$-isometry from $X$ to $Y$, then $d_{G H}(X, Y)<2 \varepsilon$. 
Because of this proposition, being close enough in the Gromov-Hausdorff metric is the same as the existence of an $\varepsilon$-isometry for $\varepsilon$ small enough.

We note that an $\varepsilon$-isometry need not be continuous.

We will define a kind of inverse for an $\varepsilon$-isometry and give a few lemmas whose proofs we omit (as they are straightforward). For an $\varepsilon$-isometry $f: X \rightarrow Y$, we call a function $f^{-1}: Y \rightarrow X$ an inverse of $f$ if $d_{Y}\left(y, f\left(f^{-1}(y)\right)\right) \leq \varepsilon$ for $y \in Y$. Such functions obviously exist since $f(X)$ is an $\varepsilon$-net in $Y$.

Lemma 1. If $f: X \rightarrow Y$ is an $\varepsilon$-isometry, then an inverse $f^{-1}: Y \rightarrow X$ of $f$ is a $3 \varepsilon$-isometry.

Lemma 2. If $f: X \rightarrow Y$ is an E-isometry and $g: Y \rightarrow Z$ is a $\delta$-isometry, then $g \circ f: X \rightarrow Z$ is an $\varepsilon+2 \delta$-isometry.

Corollary 1. Let $f: X \rightarrow Y$ be an $\varepsilon$-isometry and $f^{-1}$ be an inverse of $f$. Then, $f^{-1} \circ f: X \rightarrow X$ is a $7 \varepsilon$-isometry and $f \circ f^{-1}: Y \rightarrow Y$ is a $5 \varepsilon$-isometry.

\section{The Stability Theorem}

First we want to motivate our exclusion of vertices of degree 2. Consider the graphs in Figure 1:

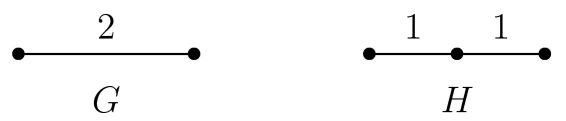

Figure 1: Isometric but non-equivalent graphs

$G$ has two vertices and an edge with weight 2 and $H$ has three vertices and two edges with weights 1 . These graphs are obviously isometric with respect to the induced intrinsic metrics, but they are not equivalent as graphs. This is due to the presence of the middle vertex of $H$ which has degree two. Another approach to circumvent this difficulty could be to allow only constant weights (say, 1, for each edge) and indeed, that would be preferable for some purposes. But we feel that the choice we made is somewhat more flexible.

Lemma 3. Let $G$ and $H$ be admissible weighted graphs with minimal weights $\geq r>0$ and $f: G \rightarrow H$ be an $\varepsilon$-isometry with $\varepsilon \ll r$. (This means that $\varepsilon$ is small enough with respect to $r$ and can be further precised to make the following proof work.) Let $a \in V(G)$ be a vertex of $G$. Then, $f(a)$ is in the $6 \varepsilon$-neighborhood of a unique vertex of $H$. (We use closed neighborhoods.)

Proof. We distinguish two cases: $\operatorname{degree}(a)=1$ and $\operatorname{degree}(a) \geq 3$. First we consider the case degree $(a) \geq 3$. In fact, we can be stiffer in this case and show that $f(a)$ is in the $3 \varepsilon$-neighborhood of a vertex of $H$. Assume to the contrary that $f(a)$ is in the $3 \varepsilon$ neighborhood of no vertex of $H$. This means that there is no vertex in the $3 \varepsilon$-neighborhood 
of $f(a)$ and thus the $3 \varepsilon$-neighborhood of $f(a)$ is a simple arc (lying in the interior of some edge) in $H$.

Consider the points $a_{1}, a_{2}, \ldots, a_{n}$ of $G$ having (exact) distance $2 \varepsilon$ to the vertex $a$ ( $n$ being the degree of $a)$. Then, by $\varepsilon$-isometry, $\varepsilon \leq d_{H}\left(f(a), f\left(a_{i}\right)\right) \leq 3 \varepsilon(i=1,2, \ldots, n)$. On the other hand, $d_{G}\left(a_{i}, a_{j}\right)=4 \varepsilon$ for $i \neq j$ and by $\varepsilon$-isometry, $3 \varepsilon \leq d_{H}\left(f\left(a_{i}\right), f\left(a_{j}\right)\right) \leq 5 \varepsilon$.

Thus, one has to place into an arc of length $6 \varepsilon$ centered around $f(a)$ at least three points, any two of which is at least $3 \varepsilon$ apart and each of which is at least $\varepsilon$ apart from $f(a)$. This is obviously not possible.

Now we consider the case $\operatorname{degree}(a)=1$.

Assume that $f(a)$ does not lie in the $6 \varepsilon$-neighborhood of any vertex of $H$. Then the $6 \varepsilon$-neighborhood of $f(a)$ is a simple arc in $H$. Let us denote the endpoints of this arc by $b$ and $c$ and let $b^{\prime}$ and $c^{\prime}$ be the points in distances $\frac{7}{2} \varepsilon$ from $f(a)$ lying in (unoriented) segments $[b, f(a)],[c, f(a)]$ as shown in Figure 2.

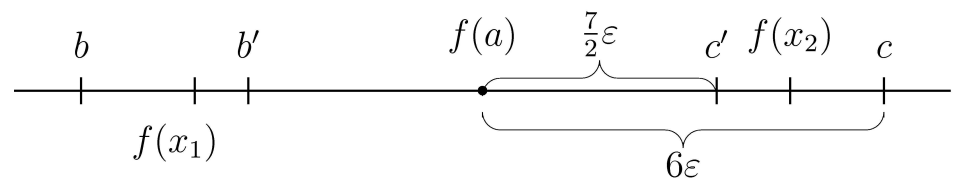

Figure 2: $6 \varepsilon$-neighborhood of $f(a)$

Now consider in $G$ the segment $\left[a, a^{\prime}\right]$ lying in the edge emanating from $a$ of length $7 \varepsilon$.

We claim that one of the segments $\left[b, b^{\prime}\right]$ and $\left[c, c^{\prime}\right]$ does not contain any point in $f(G)$. Assume to the contrary that there are points $x_{1}, x_{2} \in G$ such that $f\left(x_{1}\right) \in\left[b, b^{\prime}\right]$ and $f\left(x_{2}\right) \in\left[c, c^{\prime}\right]$. Both of these points $x_{1}$ and $x_{2}$ must belong to $\left[a, a^{\prime}\right]$, because for a point $x$ outside $\left[a, a^{\prime}\right]$ we have $d_{G}(x, a)>7 \varepsilon$ and hence $d_{H}(f(x), f(a))>6 \varepsilon$.

Now, as $d_{H}\left(f\left(x_{1}\right), f(a)\right) \geq \frac{7}{2} \varepsilon$ and $d_{H}\left(f\left(x_{2}\right), f(a)\right) \geq \frac{7}{2} \varepsilon$, it must hold $d_{G}\left(x_{1}, a\right) \geq \frac{5}{2} \varepsilon$ and $d_{G}\left(x_{2}, a\right) \geq \frac{5}{2} \varepsilon$. But then $d_{G}\left(x_{1}, x_{2}\right) \leq \frac{9}{2} \varepsilon$, hence $d_{H}\left(f\left(x_{1}\right), f\left(x_{2}\right)\right) \leq \frac{11}{2} \varepsilon$, contradicting $d_{H}\left(f\left(x_{1}\right), f\left(x_{2}\right)\right) \geq 7 \varepsilon$. Thus one of $\left[b, b^{\prime}\right]$ and $\left[c, c^{\prime}\right]$ does not intersect $f(G) \subset H$. But then the midpoint of that segment would not lie in the $\varepsilon$-neighborhood of any point $f(x)(x \in G)$, contradicting the assumption that $f$ is an $\varepsilon$-isometry.

This lemma enables us to define for an $\varepsilon$-isometry $f: G \rightarrow H(\varepsilon \ll r)$ a map

$$
\begin{aligned}
\tilde{f}: V(G) & \longrightarrow V(H) \\
a & \longmapsto \tilde{f}(a), \quad d_{H}(f(a), \tilde{f}(a)) \leq 6 \varepsilon .
\end{aligned}
$$

Since $\varepsilon \ll r$, the vertex $\widetilde{f}(a)$ of $H$ is unique.

If $f^{-1}$ is an inverse of $f$, we get a similar map

$$
\widetilde{f^{-1}}: V(H) \longrightarrow V(G)
$$

and one can show with some simple (but tedious) considerations that

$$
\widetilde{f^{-1}} \circ \widetilde{f}=\widetilde{f^{-1} \circ f}=\operatorname{Identity}(V(G))
$$


and

$$
\tilde{f} \circ \widetilde{f^{-1}}=\widetilde{f \circ f^{-1}}=\operatorname{Identity}(V(H)) .
$$

As a consequence we obtain:

Lemma 4. For an $\varepsilon$-isometry $f: G \rightarrow H \quad(\varepsilon \ll r)$ the induced map $\tilde{f}: V(G) \rightarrow V(H)$ is a bijection between the set of vertices of $G$ and the set of vertices of $H$.

We now show that given any pair $a, b$ of vertices of $G$ (which might also coincide), the set $\mathcal{E}(a, b)$ of edges between these vertices is in one-to-one correspondence with the set $\mathcal{E}(\widetilde{f}(a), \tilde{f}(b))$ of edges between the images of the given vertices under $\tilde{f}$.

We first prove the following

Lemma 5. Let $f: G \rightarrow H$ be an $\varepsilon$-isometry $(\varepsilon \ll r), a, b \in V(G), a \neq b$. If $p$ is the midpoint of an edge e between the vertices $a$ and $b$, then $f(p)$ lies on an edge $\widetilde{e}$ between $\widetilde{f}(a)$ and $\widetilde{f}(b)$.

Proof. First we note that $f(p)$ cannot be closer than $6 \varepsilon$ to any vertex of $H$. If that were the case, for example $d_{H}(f(p), \widetilde{f}(c)) \leq 6 \varepsilon$ for a vertex $c \in G$, then, since $d_{H}(f(c), \tilde{f}(c)) \leq 6 \varepsilon$ by Lemma 3 , we would get $d_{H}(f(p), f(c)) \leq 12 \varepsilon$. By $\varepsilon$-isometry of $f, d_{G}(p, c) \leq 13 \varepsilon$. On the other hand we have $d_{G}(p, c) \geq \frac{1}{2} \omega(e) \geq \frac{r}{2}$ contradicting $\varepsilon \ll r$.

This shows that $f(p)$ is an inner point of an arc. This arc must be an arc connecting $\widetilde{f}(a)$ and $\widetilde{f}(b)$. We could do the similar calculations, but we can understand the picture also qualitatively: $a$ and $b$ are the two vertices closest to $p$ and with the same distance to p. $\widetilde{f}(a)$ and $\widetilde{f}(b)$ must be the two vertices closest to $f(p)$ and with distances equal within some bounded multiplies of $\varepsilon$. This necessitates that $\widetilde{f}(a)$ and $\widetilde{f}(b)$ are the vertices of $H$ which are the endpoints of the arc on which $f(p)$ lies. At the same time, $f(p)$ is close enough to the true midpoint of this arc between $\widetilde{f}(a)$ and $\widetilde{f}(b)$.

This lemma enables us to define a map

$$
\begin{aligned}
\widetilde{F}(a, b): \mathcal{E}(a, b) & \longrightarrow \mathcal{E}(\tilde{f}(a), \tilde{f}(b)) \\
e & \longmapsto \widetilde{e} .
\end{aligned}
$$

This map is one-to-one: if we consider the midpoints $p_{1}$ and $p_{2}$ of edges $e_{1} \neq e_{2}$, they cannot be mapped into the same arc between $\widetilde{f}(a)$ and $\widetilde{f}(b)$, because then both had to lie close enough to the midpoint of that arc, consequently close enough to each other, whereas they are far apart in $G$. One can define the inverse of $\widetilde{F}(a, b)$ with the help of an inverse $f^{-1}$ of $f$, with the result that $\widetilde{F}(a, b)$ is one-to-one and onto.

A similar line of thought shows that

$$
\widetilde{F}(a, a): \mathcal{E}(a, a) \longrightarrow \mathcal{E}(\tilde{f}(a), \tilde{f}(a))
$$

is one-to-one and onto: The midpoint of an arc around $a$ must lie on an arc around $\widetilde{f}(a)$ and close to its midpoint; midpoints of different arcs around $a$ must be mapped to different arcs around $\tilde{f}(a)$. One can consider again $f^{-1}$ for surjectivity. We have thus proven the following theorem which is equivalent to Theorem 1 : 
Theorem 2. Let $G$ and $H$ be admissible weighted graphs with minimal weights $\geq r>0$ and let $f: G \rightarrow H$ be an $\varepsilon$-isometry with $\varepsilon \ll r$. Then $G$ and $H$ are equivalent graphs.

Remark 1. The following property could also be deduced from the above proof: As $G$ and $H$ become homeomorphic (under the hypotheses of Theorem 2) one can consider the Lipschitz distance $\operatorname{Lip}(G, H)$ and estimate it above via $r, \varepsilon$; more precisely, there exists a function $\Delta_{r}(\varepsilon)$ such that $\Delta_{r}(\varepsilon) \rightarrow 0$ as $\varepsilon \rightarrow 0$ and $\operatorname{Lip}(G, H)<\Delta_{r}(s)$.

In this form, the statement resembles a theorem of Gromov for Riemann manifolds with bounded sectional curvatures and injectivity radius [5, p.384],[6].

Remark 2. Recently, some metric spaces of graphs are being considered by graph theoreticians (see [1],[2],[3]). It would be interesting to consider stability questions with respect to these metrics also.

Acknowledgements. We are indebted to the referee for Remark 1.

\section{References}

[1] C. Borgs, J. Chayes, L. Lovasz, V.T. Sos, K. Vesztergombi, "Counting graph homomorphisms", Topics in Discrete Mathematics M. Klazar, J. Kratochvil,M. Loebl, J. Matousek, R. Thomas, P. Valtr, (Editors), Springer, 2006, pp. 315-371.

[2] C. Borgs, J.T. Chayes, L. Lovasz, V.T. Sos, and K. Vesztergombi, Convergent Graph Sequences I: Subgraph frequencies, metric properties, and testing, preprint, 2006.

[3] C. Borgs, J.T. Chayes, L. Lovasz, V.T. Sos, and K. Vesztergombi, Convergent Graph Sequences II: Multiway Cuts and Statistical Physics, preprint, 2007.

[4] D. Burago, Y. Burago, S. Ivanov, A Course in Metric Geometry, A.M.S., 2001.

[5] M. Gromov, Metric Structures for Riemannian and non-Riemannian Spaces, Birkhäuser, Boston, 2007.

[6] Yu. D. Burago, S.G. Malev, D.I. Novikov, A Direct Proof of one Gromov's Theorem, arXiv:0802.0098v1. 